\title{
Entre santé publique et intérêt économique
}

\section{Rainer M. Kaelin}

Dr, Spécialiste Pneumologie et Médecine interne, membre FMH

\section{Introduction}

Les maladies non transmissibles MNT (noncommunicable diseases, NCD) sont un problème majeur de santé du monde industrialisé. L'OMS [1] leur attribue 40 mio de morts par an. Pour la société agée de la Suisse, les MNT sont un poids particulier. La stratégie nationale des MNT 2017-2024 [2] indique un coût annuel de santé à 70-80 mia de CHF, dont 80\% imputables aux MNT. Cancers, maladies cardiovasculaires, respiratoires, de l'appareil locomoteur et diabète causent près de la moitié des coûts, susceptibles d'être influencés par la prévention. Cet article décrit la stratégie nationale et le défi qu'elle présente pour médecins, organisations de santé et politiciens.

\section{Stratégie nationale NCD 2017-2024 [2]}

Elaborée par l'Office fédéral de la santé publique (OFSP), elle est signée par le conseiller fédéral Alain Berset et les directions cantonales de la santé. Le plan des mesures a été signé en plus par Promotion Santé Suisse. L'avant-propos [3] donne la vision: "La stratégie MNT est axée sur des expériences [...] des programmes [...] tabac, alcool, alimentation et activité physique [...] de la confédération, des cantons, des communes, de la fon-

\section{Le pouvoir de l'Etat de modifier les conditions- cadres est traité de manière insuffisante.}

dation Promotion santé suisse, [...] ces acteurs collaboreront encore plus étroitement.» On prévoit trois mesures principales: a. promotion de la santé / prévention destinées à la population; b. prévention dans le domaine des soins; c. dans l'économie et le monde du travail. Cinq mesures transversales sont énumérées: coordination/coopération; financement; monitorage/ recherche; information/formation; conditions-cadres. La Confédération n'agira pas sur le plan législatif: «La promotion de la santé et la prévention [...] principalement planifiées au niveau cantonal et mises en œuvre par des ONG's, des villes, et des communes. Le docu- ment souligne, troisième volet, le rôle de l'économie pour la santé de la population [...]. Les mesures [...] sont prises volontairement.»

Les mesures transversales sont attribuées aux agences de la Confédération (OFSP, sécurité alimentaire, agriculture, prévention du tabagisme, alcools, SECO, douanes, etc.). Cette énumération mentionne indirectement les mesures structurelles, c.-à-d. les mesures légales qui créent le contexte, où "le bon choix devient le choix facile».

On cherche en vain une identification des mesures de la prévention comportementale (axées sur le comportement de la population et des acteurs) et de la prévention par modification des conditions-cadres (mesures structurelles qui modifient le contexte). Cette prérogative de l'Etat est traitée de manière insuffisante. Aucun objectif législatif est identifié. Il est uniquement dit que «les mesures de prévention structurelle sont nombreuses. [...] pistes cyclables, [...] espaces naturels et de détente, une offre de repas équilibrés dans les cantines des lieux de travail et des écoles. [...] L'économie aussi [...] contribue soit par un code de comportement ou en s'imposant les règles pour une composition saine des produits alimentaires (avec moins de sucre, de sel, ou moins de graisses) ou en encourageant les employés à bouger plus. Des règles légales se sont révélées particulièrement efficaces, [...] la fixation des prix, les conditions d'achat, la publicité et l'impôt sur des produits et services nocifs à la santé, comme l'alcool, les cigarettes, les jeux de hasard.» Ceci reste vague. Les lacunes légales de la protection contre la fumée passive et la LPTab (baisse du taux des fumeurs par l'interdiction de la publicité) ne sont pas mentionnées.

\section{Les lacunes}

La stratégie touche tous les aspects préventifs des MNT. Les experts de l'OFSP souslignent coordination et suivi scientifique. Celui-ci, cependant, montrera tôt les lacunes de la stratégie:

- activités et initiatives uniquement cantonales/locales 
- absence d'objectifs chiffrés d'une baisse du taux d'obèses et de fumeurs

- absence d'objectifs législatifs fédéraux

- absence d'analyse des projets de loi échoués, pour trouver une stratégie à faire adopter une prévention structurelle par majorité parlementaire

Influencés par des cercles économiques, de toute évidence, à l'OFSP les experts ont prévalu qui optimisent le statu quo, mais qui n'ont pas d'objectifs plus ambitieux. Ce qui est démontré par la mise en consultation de la stratégie [4]: deux tiers des réponses approuvent la stratégie, tout en critiquant le manque de prévention structurelle. Le fonds de prévention du tabagisme a motivé son refus par ce manque. Un quart des répondants, tous dans la catégorie économie et assureurs, ont refusé, ce qui est cohérent. Car, suivant l'USAM et economiesuisse, les parlementaires alliés ont bloqué ou dénaturé tous les projets de la prévention des années passées, mêmes modérés: loi sur la prévention, loi sur l'alcool, LPTab, loi fédérale-alibi de protection contre la fumée passive.

\section{Santé publique}

Malgré les expériences passées [5], les experts de l'OFSP n'ont pas thématisé l'attitude hostile de l'économie à l'égard de la prévention. Ils ont élaboré une stratégie qui évite le conflit par la mise en parenthèse de la prévention structurelle.

Des efforts importants de communication sont donc nécessaires. La prévention structurelle, son efficacité prouvée et son apparente incompatibilité avec l'économie sont susceptibles d'être expliquées aux fonctionnaires, à la population et aux parlementaires [5]. Ceci brisera le préjugé contre "la prévention ordonnée par l'Etat». Cette langue de bois de l'USAM et d'economiesuisse a créé depuis 2008 la confusion entre prévention comportementale et structurelle. Par une communication différenciée, la pesée d'intérêts entre avantage particulier et intérêt de santé de la population sera amenée à une décision politique transparente, loin de "prévention = atteinte à la libérté». Devant le conflit d'intérêts inévitable, l'OMS met en garde: «Non-State actors (of public health) include academia, and relevant nongovernmental organizations, as well as selected private sector entities, as appropriate, excluding the tobacco industry" [6]. Le comportement de l'industrie du tabac et des boissons, ainsi que de ses alliés parlementaires, est incompatible avec la prévention. Leur attitude négative s'était imposée uniquement dans le contexte d'une opinion publique désinformée.
La communication de Santé Publique Suisse a ignoré la prévention structurelle depuis longtemps, favorisant la désinformation du public. A ses congrès annuels, ni le tabac ni l'étiquetage des denrées alimentaires n'a jamais été un sujet. L'appel des médecins adressé à Santé Publique Suisse en 2016, de se positionner publiquement dans le débat sur la LPTab, est resté sans réponse [7]. La stratégie MNT lance le défi à la santé publique, comme à tous les médecins experts, de sortir enfin du silence [8-10] et de contribuer à une opinion publique.

\section{Le corps médical}

Sans mentionner la prévention structurelle, les articles du BMS sur la stratégie nationale MNT [11] décrivent la tâche des médecins [12]: intégrer la prévention dans la prise en charge des patients, dans la formation et la formation continue. L'importance du médecin dans l'«interprofessionnalité» est soulignée. Le Dr Quinto, responsable de la prévention à la FMH, nomme les objectifs de l'organisation faîtière [13]: a. promotion de santé, prévention et diminution de la charge des maladies; b. rôle central en santé publique au plan national; c. participation active du corps médical pour sa réalisation dans le quotidien. C'est abstrait. Cependant, il interpelle concrètement la politique concernant l'inégalité sociale: «Une bonne politique de la formation et de la santé pourraient réduire [ces inégalités] si d'autres domaines de la politique tenaient compte du problème»; et: "On ne peut pas demander de plus en plus [...] de se responsabiliser et d'autre part se rendre responsable sur le plan politique d'une prévention structurelle faible et arriérée. Il n'est pas éthique de dire 'tu ne devrais pas fumer', mais [...] pour des raisons commerciales continuer à promener devant le nez des jeunes des cigarettes lors d'évènements et manifestations.»

Experts médicaux et de santé publique disposent aussi d'arguments économiques pour convaincre de la valeur

\section{La communication de Santé Publique Suisse a} ignoré la prévention structurelle depuis longtemps, favorisant la désinformation du public.

de la prévention structurelle [14]: les coûts directs des maladies cardiovasculaires et des maladies respiratoires se montent à >10 mia, resp. >1,5 mia de CHF/an Les coûts indirects (perte de productivité par arrêts maladie, etc.) se chiffrent à >5-6,5 mia, resp. 0,5-3 mia de $\mathrm{CHF} / \mathrm{an}$. Cela sera moins avec un quota de fumeurs réduit par l'interdiction de la publicité dans la LPTab. L'OFSP le dit: «Investir 1 franc dans la prévention du tabagisme en économise 41 dans les coûts.» 


\section{Politiciens de la santé}

Le 14 décembre 2016, les conseillers des Etats Hans Stöcklin et Joachim Eder, et la conseillère nationale Ruth Humbel fondèrent le groupe MNT avec 34 parlementaires pour ancrer les MNT dans la politique. Ces politiciens devront convaincre leurs collègues. Car de toute évidence ceux-ci favorisent l'industrie au détriment d'intérêts supérieurs. L'argumentation «Nous voulons la protection de la jeunesse; nous refusons l'interdiction de la publicité», employée contre toute bonne foi pour retourner le projet de la LPTab, montra ce que les MNT leur signifient. En février 2017, le Conseil des Etats [15] approuva le «cadeau de Noël» du Conseil

\section{L'Etat renonce à des gains fiscaux, tout en permettant à l'industrie de majorer ses marges.}

national [16]: on retira au gouvernement la compétence de freiner la consommation de tabac par la hausse des taxes (une augmentation de $>10 \%$ est efficace à cet effet). Depuis la dernière hausse de 2013, les cigarettiers montèrent les prix par petits pas, sans freiner la consommation. Donc: l'Etat renonce à des gains fiscaux, tout en permettant à l'industrie de majorer ses marges. Combattre les MNT signifie lutter contre l'épidémie mondiale du tabagisme, comme le préconise la CCLAT. Aucun parlementaire de la majorité n'a mentionné cette convention pendant la législature.

\section{Conclusions}

Le défaut fatal de la stratégie MNT 2017-2024 est le manque de mesures structurelles nationales. Par cela, le papier esquive le conflit d'intérêt incontournable entre l'industrie du tabac / alimentaire et une prévention efficace. Car les mesures structurelles cantonales, les mesures "volontaires», ainsi que l'«autocontrôle» de la publicité se révéleront des feuilles de vignes futiles en face des contre-stratégies globales du marketing de ces industries.

Dans le contexte hostile, les médecins, les experts en prévention, les organisations de santé et le groupe parlementaire MNT sont mis au défi de convaincre par des arguments scientifiques. Ils expliqueront aux gens et aux parlementaires les relations causales entre conditions-cadres, style de vie et le développement des MNT. Les formules "Responsabilité au lieu de tutelle», «Publicité est information du consommateur» ou «Un produit légal a droit à la publicité» sont à mettre en ques- tion aussi par les média. Médecins et experts en santé avec des parlementaires motivés vont former par une communication transparente une opinion publique que le parlement ne pourra plus ignorer. Alors, la prévention structurelle ne sera plus discutée comme politique de droite ou de gauche, mais comme une question d'honnêteté intellectuelle: si l'intérêt particulier des industries du tabac / alimentaire, qui sont (co-)responsables du problème de santé publique du tabagisme / de la nutrition, serait supérieur à celui de la santé de la population. Public Health works, if there is political will. La stratégie MNT 2017-2024 met au défi la volonté politique de tous les professionnels de la santé. Le débat parlementaire au sujet de la LPTab révisée et la ratification de la CCLAT constitueront le premier test de cet effort interprofessionnel destiné au succès.

\section{Références}

1 WHO global status report on noncommunicable diseases. Fact sheet, updated 2017. http://www.who.int/mediacentre/factsheets/ fs355/en/

2 OFSP et Conférence suisse des directrices et directeurs cantonaux de la santé: Les maladies non transmissibles: un défi. Stratégie nationale Prévention des maladies non transmissibles 2017-2024. (stratégie MNT), version courte. Avril 2016. www.bag.admin.ch

3 Plan des mesures. Stratégie nationale Prévention des maladies non transmissibles (stratégie MNT) 2017-2024. www.bag.admin.ch 4 OFSP et Conférence suisse des directrices et directeurs cantonaux de la santé: Rapport des résultats de la consultation sur la Stratégie nationale Prévention des maladies non transmissibles (stratégie MNT) 2015. www.bag.admin.ch

5 J. Cornuz, B. Burnand, I. Kawachi, F. Gutzwiller, F. Paccaud: Why did Swiss citizens refuse to ban tobacco advertising?. Tob. Control 1996;5:149-53.

6 WHO action plan for the prevention and control of noncommunicable diseases, 2013-2020.

7 R. M. Kaelin et 88 cosignataires: Lettre ouverte à Santé Publique Suisse; Bull méd. suisses 2016:97(21);741.

8 R. M. Kaelin: Prévention est affaire des méedecins - une communication transparente en est le fondement. BMS 2013;94(31-32): 1180-3.

9 R. M. Kaelin: Le silence des experts médicaux. II. La responsabilité publique des médecins dans la prévention du tabagisme; III. La responsabilité publique des médecins au sujet de l'initiative "Protection contre le tabagisme passif». Bioethica Forum. 2015;8(3):70-2; 2015;8(4):135-7.

10 R. M. Kaelin: Schweigen zur Initiative «Schutz der Bevölkerung vor Passivrauch». Zur öffentllichen Verantwortung der Aerzte - Teil 2. BMS. 2015;96(19):700-2.

11 E. Bruhin: Stratégie contre les maladies non transmissibles. BMS 2016;97(48):11672-3.

12 C. B. Quinto, B. Weil, et al.: Le rôle du corps médical dans le deuxième bloc de travail MNT. BMS 2017:98(4):100-2.

13 Spectra. 18.1.2017: Interview mit Dr Carlos Beat Quinto. www.spectra-online.ch

14 Nationale Präventionsprogramme: Studie präsentiert erstmalige Berechnungen der direkten und indirekten Kosten der wichtigsten nichtübertragbaren Krankheiten. Bulletin BAG/OFSP 1.9.2014.

15 Pressemitteilung vom 28.2.2017. ATS

16 Conseil national, session hiver 2016, douzième séance, 14.12.2016. Loi sur l'imposition du tabac. Modification. Objet 16.051 www. admin.ch/Parlement/conseil national 